\title{
Electric Vehicle with Charging Facility in Motion using Wind Energy
}

\author{
S.M. Ferdous ${ }^{2, *}$, Walid Bin Khaled ${ }^{1}$, Benozir Ahmed ${ }^{2}$, Sayedus Salehin ${ }^{1}$, Enaiyat Ghani \\ Ovy ${ }^{1}$ \\ ${ }^{1}$ Department of Mechanical \& Chemical Engineering \\ Islamic University of Technology, Board Bazar, Gazipur-1704, BANGLADESH \\ ${ }^{2}$ Department of Electrical \& Electronic Engineering \\ Islamic University of Technology, Board Bazar, Gazipur-1704, BANGLADESH \\ *Corresponding author.Tel: +8801912669030,E-mail: tanzir68@gmail.com
}

\begin{abstract}
The main disadvantage of Electric Vehicle is the lack of capability of storing sufficient energy to run the vehicle for a long time. The energy storage capacity of battery used in electric vehicle is very low compare to conventional fuels used in modern automobiles. The operation, performance and efficiency of motor driven electric vehicles are much better than engine driven vehicles, at the same time electric vehicles are very much environment friendly. Still electric vehicles are falling behind in the automobile industries due to the problem of storage of energy. This paper is based on the concept of charging the batteries of an electric vehicle when it is in motion or propelling. This may be done by using the energy of wind which is caused by the relative motion between the vehicle and the wind surrounding it. Wind turbines can be mounted on the body structure of the vehicle to generate electricity in such a way that it must not create any additional drag force (rather than the existing drag force due to frontal area and skin friction) upon the vehicle. An elaborate aerodynamic analysis of the structure of the vehicle along with the flow pattern and wind turbine is presented in the paper. Some techniques and methods are proposed to minimize the drag imposed by the introduction of the turbines as much as possible. Optimum values of different design parameters and rated velocity of the vehicle are of prime concern. With this concept it may be possible to increase the mileage of an electric vehicle up to $20 \%-25 \%$ and it will also save the charging time of the battery to a great extent. Flow pattern over the vehicle is simulated using software called ANSYS CFX.
\end{abstract}

Keywords: Electric Vehicle, Wind Energy, ANSYS-CFX, Wind Turbine, Drag Reduction

\section{Introduction}

When a vehicle moves it experience wind resistance which are classified in two different forms- frictional drag and form drag. Frictional drag arises due to viscosity of air and form drag arises due to variation of air pressure in the front and rear side of the vehicle [1]. As the vehicle moves forward, it lefts the air stream behind. A turbulence or disturbance is created on the wind when a vehicle moves through it. If stationary wind turbines are placed near the road then energy can be extracted from the wind stream generated due to the movement of the vehicle. Such a study had been carried out in University of Arizona by a group of students. If it is possible to capture those wind streams within the vehicle itself then it $\mathrm{c}$ an be used to recover some of energy that has been used to overcome the form drag (aerodynamic drag) of the vehicle. If this wind energy is used to extract some power in such a way that it does not create any component of force or thrust opposite to the direction of the propulsion of the vehicle, then this gained energy can be used to produce electricity to charge up the battery of the electric vehicle itself. At the same time drag can be expected to be reduced by passing this air to the rear side (Low pressure side) of the vehicle. Air stream sliding over the body of the vehicle cannot enter into the rear side due to vortex shedding [1]. If air streams are allowed to flow in this region by any means then the form drag will be reduced by some amount and at the same time it may be possible to generate electricity using the kinetic energy of wind. Several studies had been carried out in this field but none of them are proved to be scientific. During the Second World War, wind turbines are used in submarines to charge up the batteries when they remained static and float in the water. At present it is also common to use turbines in ships, caravans and vehicles when they are parked. But to extract power from a 
moving vehicle is quite difficult as the turbine will act as a load for the vehicle. Most of the design showed that the turbines are placed over the vehicle roof without considering the fact that it will impose an additional load for the vehicle and on the other hand no measures had been taken to reduce it. A design by Rory Handel and Maxx Bricklin showed that it has four tactically placed air intakes which will channel the air flow over the car's body towards the turbine. No such detailed design was available.

In this paper the topic is dealt by considering all the scientific facts and laws of energy conversion. A new approach is proposed and simulation of the design is carried out to analyze the behavior of the model. Some theoretical formulas have been used for the purpose of calculations.

\section{Basic Theory}

It is assumed that the vehicle is moving in a calm and steady wind stream with zero wind velocity. If the vehicle is moving at a constant speed of $15 \mathrm{~m} / \mathrm{s}(54 \mathrm{~km} / \mathrm{h})$, then we can think a wind stream with $15 \mathrm{~m} / \mathrm{s}$ is flowing around the vehicle. Normally this wind will cause a drag force which is opposite to the direction of the propulsion of the vehicle. At constant speed (zero acceleration) the energy requirements to move the vehicle forward are -To overcome the frictional force (rolling resistance of road) and to overcome wind resistance [1]. At this Condition, if the air stream flowing around the vehicle (which was not interacting with the vehicle previously) is allowed to enter inside and let it flow down to the rear side; then it may be possible to use these air streams to generate power. The vehicle has already interacted with this wind and it deflects the stream of wind at the two sides of it by stagnation at the front. This is the energy that had been lost from the vehicle to overcome the aerodynamic resistant. Now if these stream generated by the interaction of the wind and vehicle is captured within the vehicle in such a way that it would not impose an additional drag at the direction of propulsion of the vehicle, some of the energy can be recovered and fed back to the battery by means of conventional energy conversion processes. Placing a wind turbine can serve the purpose. At the same time it will help to increase the pressure at the back side (according to Bernoulii's equation pressure will be increased if velocity is decreased and velocity will be reduced at the back side of the turbine after energy extraction) which will reduce the drag force that existed before with the conventional design of the vehicle. So, vortex shedding will be reduced at the rear side. For this it is necessary to modify the design of a vehicle which gives provision of air flow through the vehicle. On the other hand positioning of the turbines will also be important because they must be placed in such a way that they do not impose or create any additional drag on the vehicle. Symmetrical positioning of the turbine can do the trick as the thrust acting on the turbines will cancel each other.

\section{Design and Modeling}

We can consider a vehicle which is redesigned to allow airflow and wind turbine can be set up to extract energy. Wind turbines are set in parallel with the flow of air. This set up will not create any additional thrust at the direction of propulsion. Two basic equations will be needed to explain the air flow and power extraction.

The air flow through the vehicle is given by, [2]

$$
\mathrm{Q}=\mathrm{C}_{\mathrm{v}} \mathrm{A} \mathrm{v}
$$

Where, $Q=$ flow rate in cubic meter per second.

$\mathrm{C}_{\mathrm{v}}=$ opening effectiveness 
[Value for $\mathrm{C}_{\mathrm{v}}$ is 0.5 - 0.6 for perpendicular flow and $0.25-0.35$ for skewed flow] [2]

$\mathrm{A}=$ Area in square meter

$\mathrm{v}=$ air velocity in $\mathrm{m} / \mathrm{s}$

This equation (1) will determine the amount of air flow through the vehicle inlet area.

Output power from a wind turbine is given by [4],

Where,

$$
\mathrm{P}_{\mathrm{T}}=0.5 \mathrm{C}_{\mathrm{P}} \rho \mathrm{Q} \mathrm{v}^{2}
$$

$\mathrm{P}_{\mathrm{T}}=$ Power output from the turbine in watt.

$\mathrm{C}_{\mathrm{p}}=$ Power co-efficient

(Assuming, $\mathrm{C}_{\mathrm{p}}=0.4$ for the design) [4]

$\rho=$ air density; $1.225 \mathrm{~kg} / \mathrm{m}^{3}$.

$\mathrm{Q}=$ air flow in $\mathrm{m}^{3} / \mathrm{s}$.

$\mathrm{v}=$ air velocity in $\mathrm{m} / \mathrm{s}$.

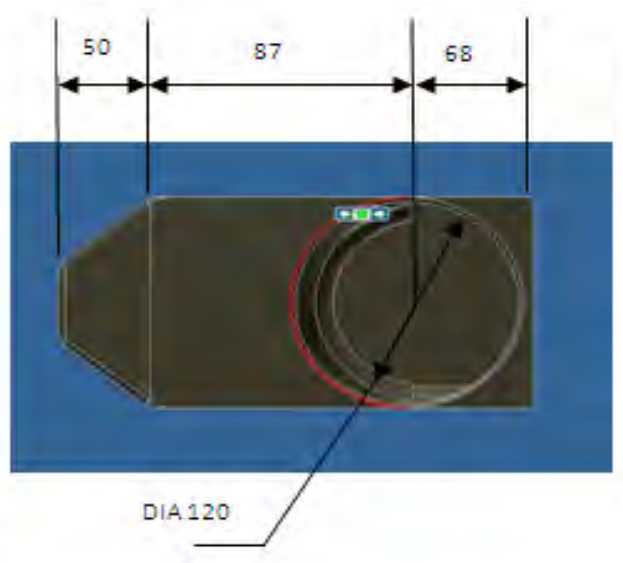

(a) Side vievs

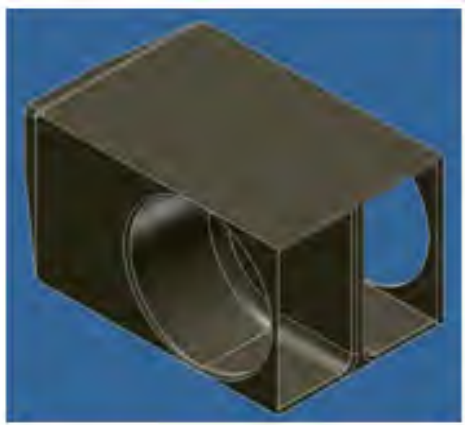

(d) 3D vievi from Back side

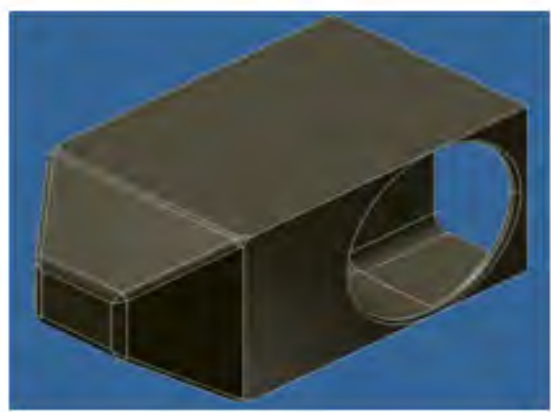

(g) 3D vievs from side

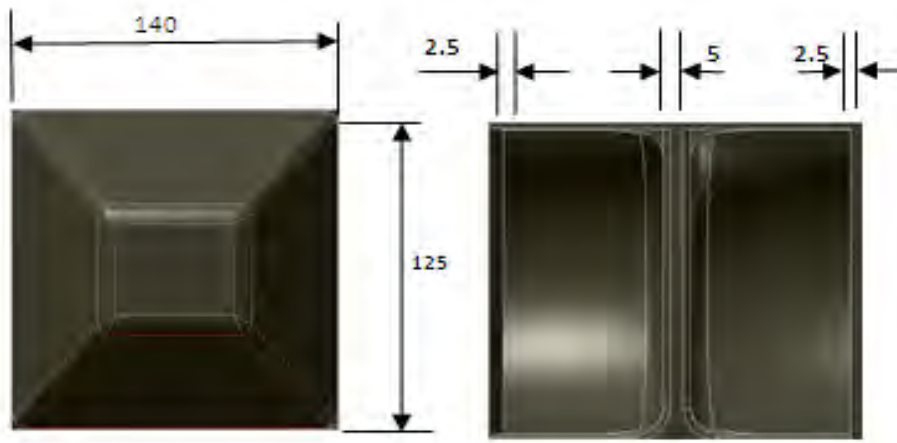

(b) Front vieve

(c) Back viev/

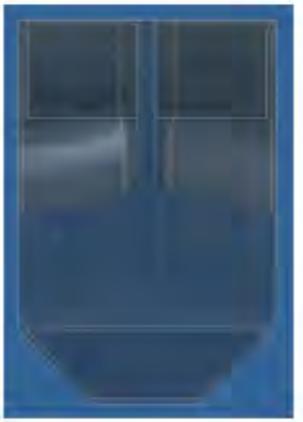

(f) Top vievs showing interior

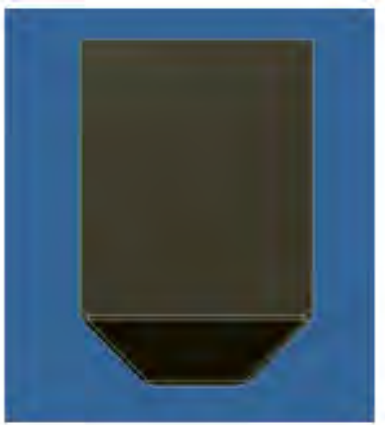

(e) Top vievs

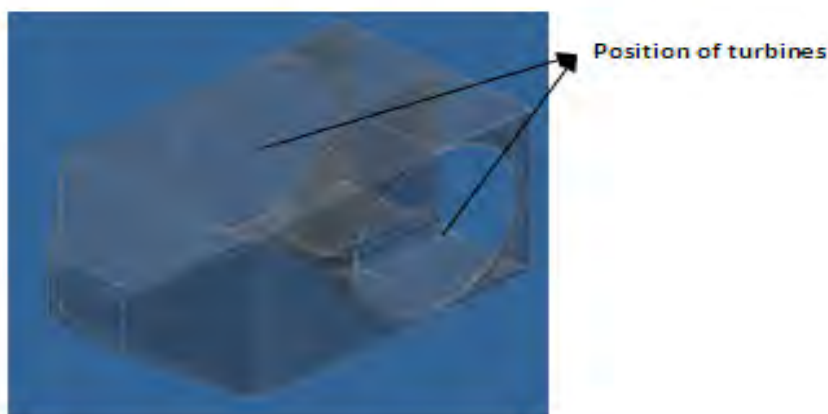

(h) 3D vievs from side shovsing the interiors

Fig. 1. 3D and isometric views of the model. The diameter of the turbine is $120 \mathrm{~cm}$ which is placed at the rear side of the vehicle. The length of the vehicle is $255 \mathrm{~cm}$. All dimensions in the diagram are in centimeters. 
In conventional vehicle air cannot go to rear side of the vehicle due to presence of boundary layers and vortex shedding. If a high-pressure and a low-pressure region can be connected via a neutral zone, then air can flow in between these pressure regions. Our design will allow the air to flow in this manner. The detail designs with dimensions are shown in the fig. 1.

The vehicle is run by a $1.5 \mathrm{KW}$ DC motor which has five $12 \mathrm{~V}, 120 \mathrm{~A}-\mathrm{h}$ D.C battery to supply power to the motor. The vehicle has to move at a velocity of $54 \mathrm{~km} / \mathrm{h}$ i.e. $15 \mathrm{~m} / \mathrm{s}$. The design of the vehicle is shown here with all dimensions. In this design the wind turbines are set in such a way so that the axial thrusts on the turbines are $180^{\circ}$ apart to each other which results in the cancellation of two thrusts. In this way this symmetrical positioning of the turbines will create no additional drag component over the vehicle. Placing the turbines on the top will increase the frontal area as well as the drag acting on the vehicle. So that approach is not scientific. Rather some solar panels can be placed on the top to aid the recharging of the vehicle, both in motion and parked position. In addition if the vehicle is parked in a place where the wind velocity is above cut in speed then it is possible to charge the vehicle and thus it could aid the total charging system and hence charging time can be reduced.

\section{Wind turbine}

The wind turbine chosen for power generation has the features stated bellow [5] -

* Two blades (for low solidity).

* Horizontal axis.

* Lift type.

* High lift to drag ratio with efficiency ranging from 0.4 to 0.45 . They need a relatively high tip speed ratio $\left(\lambda=\omega \mathrm{R} / \mathrm{v}_{\mathrm{w}}\right)$. For our design we have chosen $\lambda=6$. For this value of $\lambda$ it can be assumed that the value of $\mathrm{C}_{\mathrm{p}}$ will be 0.4 to 0.45 .
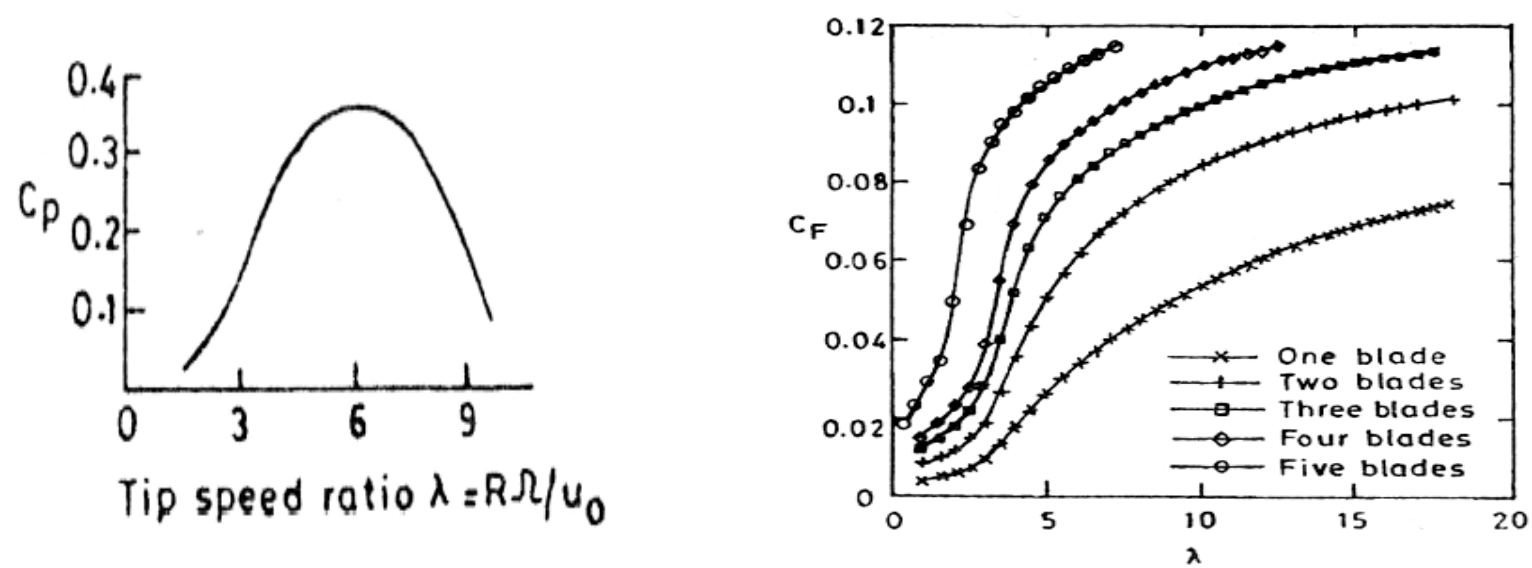

Fig. 2: Power Coefficient and Axial Thrust Coefficient for HAWT [6].

From $\mathrm{C}_{\mathrm{p}}-\lambda$ and $\mathrm{C}_{\mathrm{F}}-\lambda$ curve we can see $\mathrm{C}_{\mathrm{p}}=0.4$ and $\mathrm{C}_{\mathrm{F}}=0.055$ [6]

Where,

$\mathrm{C}_{\mathrm{F}}=$ axial thrust co-efficient.

So, $\quad \mathrm{C}_{\mathrm{p}} / \mathrm{C}_{\mathrm{F}} \approx 7$

This implies that as at perfect dynamic matching generated power will be greater than the power spend due to thrust. In other words the generated power by a turbine will be greater 
than the thrust acting on the blade as an aerofoil section has high lift to drag ratio. On the other hand, turbines are placed in parallel to the flow rather than perpendicular to the flow.

\section{Generator}

We want to use an A.C. generator with 3- $\Phi$ windings with increased no. of poles. The poles will be permanent magnets and the no. of poles will be 8 .

$\lambda=6=\omega \mathrm{R} / \mathrm{v}_{\mathrm{w}} ; \quad \omega=6 \mathrm{v}_{\mathrm{w}} / \mathrm{R}=\frac{6 \times 15}{0.6}=150 \mathrm{rad} / \mathrm{s} ; \quad$ R.P.M, $\mathrm{N}=\frac{60 \times \omega}{2 \pi}=1433.12 ;$

This eliminates the need of a gear box in the system.

We shall use a three phase A.C. to D.C. converter to charge the batteries. Cúk converter is used to give a constant $60 \mathrm{~V}$ at the output. The current of the converter will vary with the variation of the speed of the vehicle or the r.p.m of the turbine keeping the terminal voltage fixed.

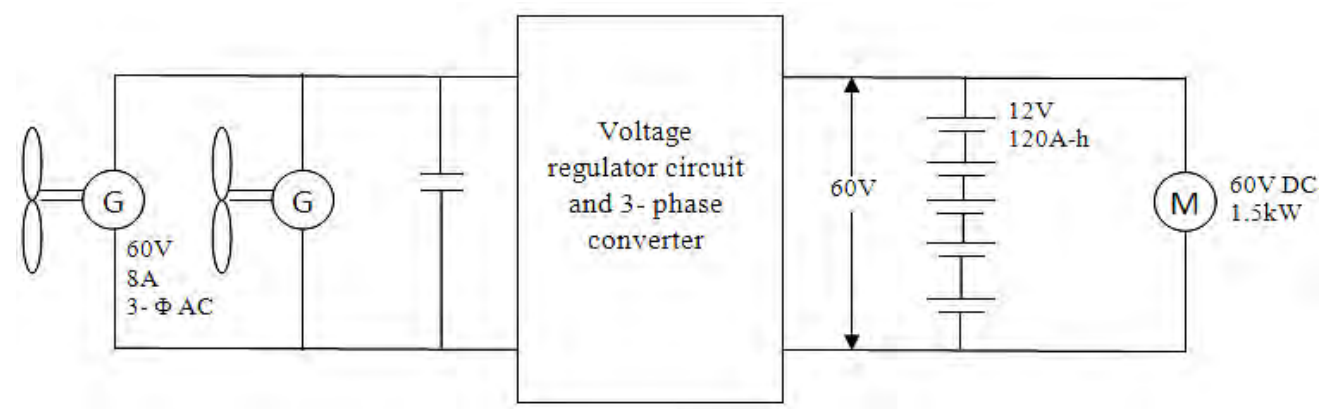

Fig. 2. Charging and control circuit of the battery. $3 \Phi$ windings are used to reduce the ripples. $A$ motor control circuit can be used to control the motor and it will also introduce the provision of Regenerative Braking. Simple Power diodes can be used for designing the converter circuit. The cut-in velocity (minimum wind velocity to generate power) of the turbines is $5 \mathrm{~m} / \mathrm{s}$.

\section{Calculation}

Using equation (1) we can calculate the amount of air flow, $\mathrm{Q}=\mathrm{C}_{\mathrm{v}} \mathrm{A} \mathrm{v}_{\mathrm{v}}=0.25 \times 0.8 \times 1.131 \times 15 \times 2=6.8 \mathrm{~m}^{3} / \mathrm{s}$

Here, $\quad \mathrm{A}=\pi \mathrm{r}^{2}=3.1416 \times 0.6^{2}=1.131 \mathrm{~m}^{2}$

$$
\mathrm{v}=54 \mathrm{kmph}=15 \mathrm{~m} / \mathrm{s}
$$

Here multiplier of $\mathrm{C}_{\mathrm{v}}$ is 0.8 as ratio of the inlet and outlet area is $1.38 . \mathrm{C}_{\mathrm{v}}$ is chosen as 0.25 as it is a skewed flow [3].

So, Power, $\mathrm{P}_{\mathrm{w}}=\frac{1}{2} \rho \mathrm{Qv}^{2}=\frac{1}{2} \times 1.2 \times 6.8 \times 15=918 \mathrm{~W}$

Assuming, $\mathrm{C}_{\mathrm{p}}=0.4$ Then we have,

$$
\mathrm{P}_{\mathrm{T}}=918 \times 0.4=367.2 \mathrm{~W} \approx 360 \mathrm{~W}
$$

So, each turbine will produce a power of $180 \mathrm{~W}$. This much power will be fed back to the battery when it is moving at a constant velocity of $15 \mathrm{~m} / \mathrm{s}$.

So increase in mileages $=(1500-1140) / 1500 \times 100 \%=24 \%$. This is due to the feeding back of some of the energy captured by the turbine which is spend to overcome the aerodynamic drag. That means the turbines are capturing some fractions of the energy which has already been spend by the vehicle to overcome the aerodynamic drag. 


\section{Simulation Result}

The Flow pattern over the model is simulated using ANSYS CFX. Two models had been chosen for simulation. One is the conventional design and another one is modified design which includes turbines on the vehicle. Using the simulation result the pressure difference and force acting in the direction of flow (i.e. the thrust acting against the direction of propulsion) is calculated. The simulation results are shown and analyzed in the following figures.
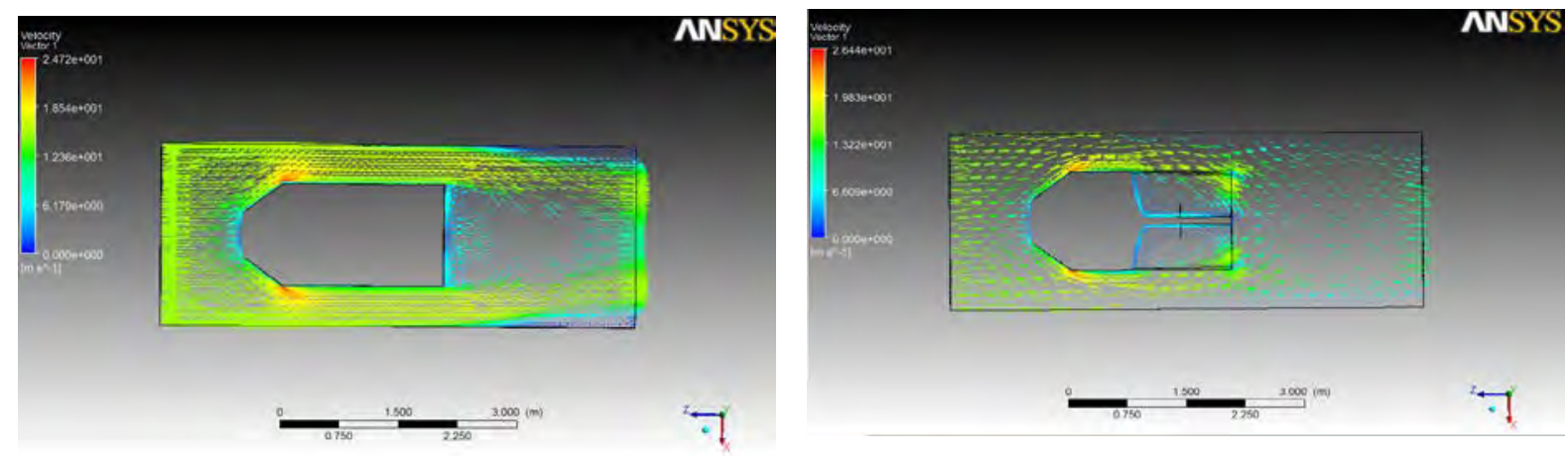

(a) Conventional vehicle model without ducts for turbine

(b) Modified vehicle model with ducts for turbine

Fig. 3. Flow pattern around the vehicle using the velocity vectors. Wind is entering inside the vehicle and going out. Energy will be extracted from this flow.

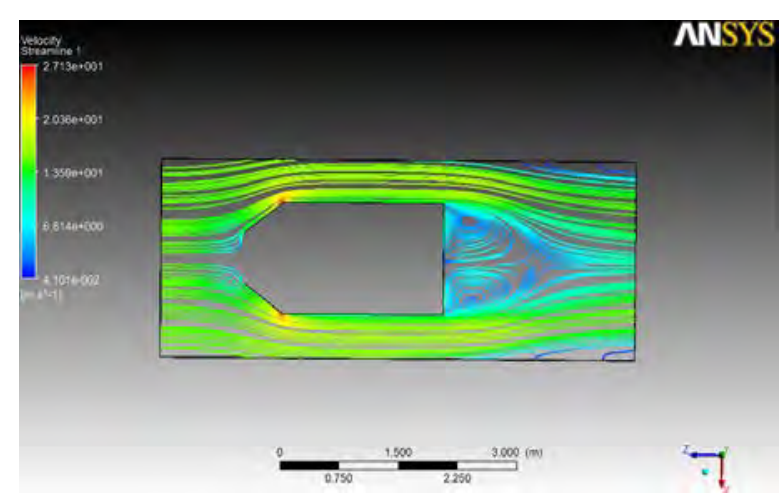

(a) Conventional vehicle model without ducts for turbine

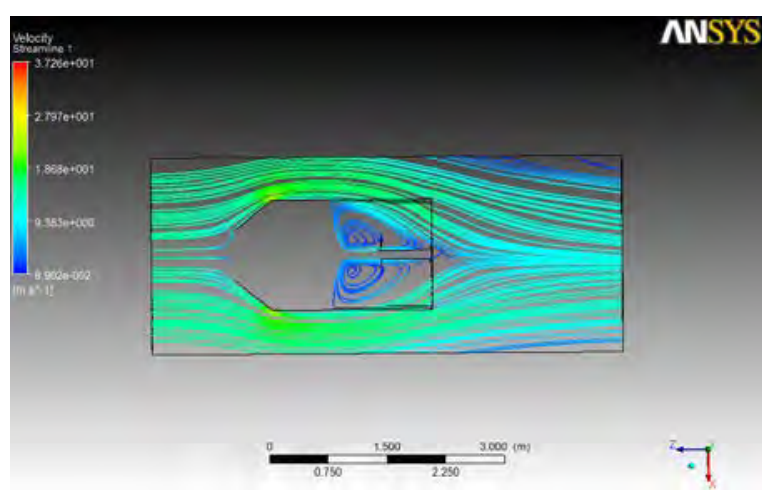

(b) Modified vehicle model with ducts for turbine

Fig. 4. Streamline of flow over the two different models of the vehicle. It may be noted that the vortexes on the modified design is reduced. On the other hand an additional propulsive thrust can be obtained as the streamlines are leaving the vehicle.

Analyzing figure 3 and 4, it can be seen that the wake region and vortexes are reduced for the modified design which implies that the force (form drag) that existed before is reduced. So it can be concluded that the prediction of flow through the duct and hence reduction of drag should be possible with this modified design. On the other hand, turbines can be placed in these ducts for extraction of some of the kinetic energy contained in the flow. The inlet and outlet pressure along with forces of these two models found from simulation are tabulated in Table 1. 
Table 1. Simulation results for pressure and forces

\begin{tabular}{ccccc}
\hline $\begin{array}{c}\text { Comparison } \\
\text { Parameters }\end{array}$ & \multicolumn{2}{c}{ Conventional Design } & \multicolumn{2}{c}{$\begin{array}{c}\text { Modified Design with ducts } \\
\text { for turbine }\end{array}$} \\
& Inlet & Outlet & Inlet & Outlet \\
\hline Pressure(Pa) & 2.29185 & -1.24196 & 2.28742 & 0.240414 \\
Force(Drag)(N) & 45.9144 & 6.5354 & 15.5202 & 11.605 \\
\hline
\end{tabular}

Table. 1 shows the plane force and pressure over the two designs have been tabulated. The variation of force and pressure are identical for the both designs, but there are variations in the magnitudes of the parameters. The outlet pressure is increased for the modified design which indicates a reduction in form drag.

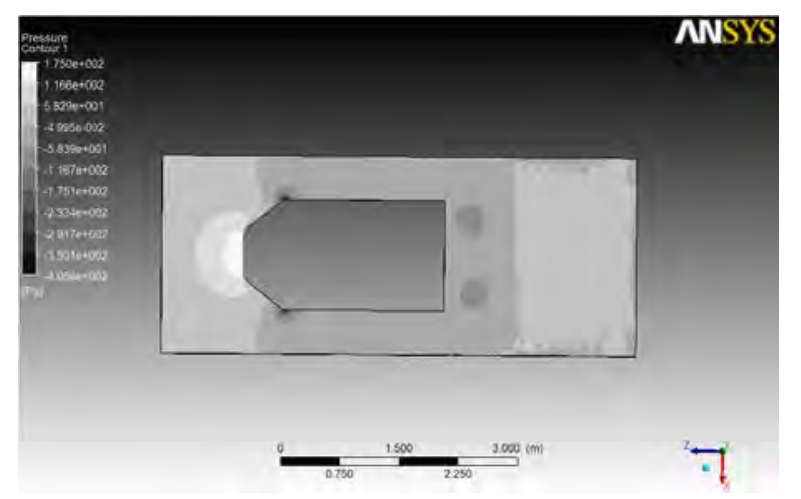

(a) Conventional vehicle model without ducts for turbine

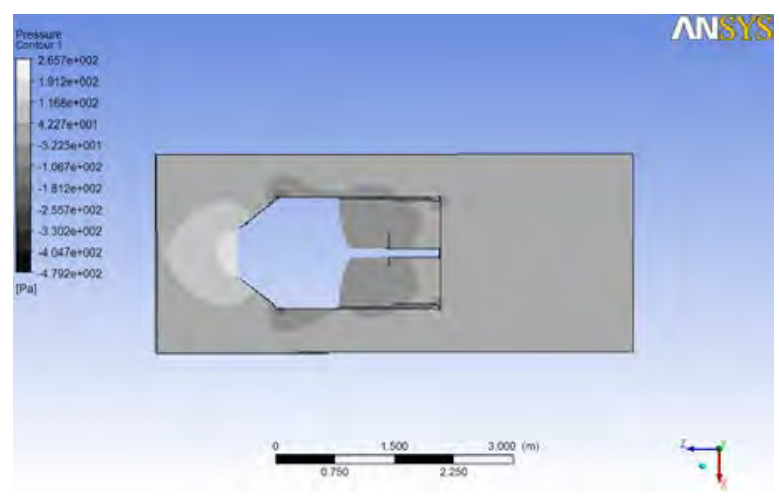

(b) Modified vehicle model with ducts for turbine

Fig. 5. Pressure contour around the two models. These figures indicate that the variation of pressure and generation of force due to this pressure variation would be identical but opposite in direction. This symmetry in the design should cancel out the additional thrust crated on the vehicle.

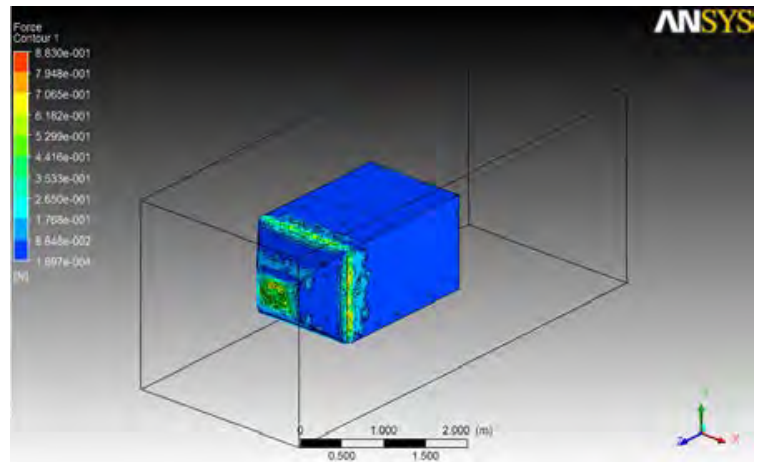

(a) Conventional vehicle model without ducts for turbine

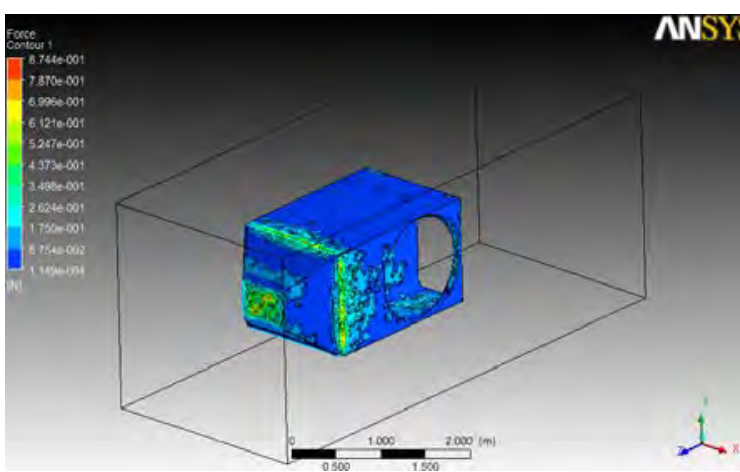

(b) Modified vehicle model with ducts for turbine

Fig. 6. Force contour showing force exerted by the air on the two vehicles which are almost same.

Further analyzing the diagrams we can see (from Fig.3) that the thrust will not be on the axis of the turbine. That means an additional drag force will arise due to placement of the turbines. We predicted that the thrust will be 180 degree apart and hence cancel out each other. But 
from velocity vectors we found that the thrusts are not fully at opposite rather they are in a skewed direction. The horizontal components will cancel each other but the vertical components will impose a resultant force. Hence a resultant thrust will be generated against the direction of propulsion on the turbine.

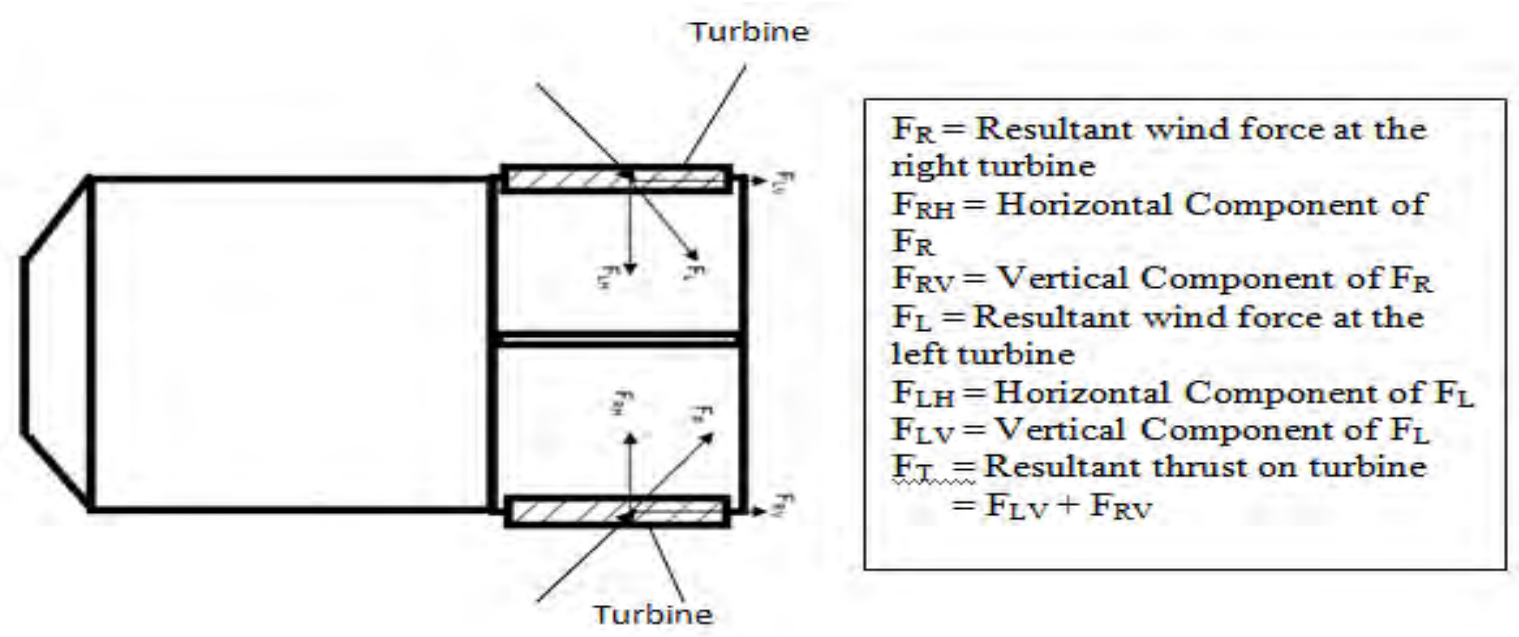

Fig. 8: Resultant thrust generated on the turbines due to air flow through the ducts.

\section{Conclusion}

The prime concern with this model is that whether this design will create any additional resistive force components opposite to the direction of the propulsion. It has been found by the simulation that a drag will be induced due to addition of turbine. Overall simulation result along with graphs from Fig. 3 will suggest that the overall effect will be same which means the modified design will experience almost same amount of drag compare to the conventional one. But the addition of turbines may give the provision of capturing some energy which will offer some benefits for the vehicle as discussed earlier. A physical structure of the design should be used to carry out wind tunnel tests which are yet to be done. At first the system may resembles with perpetual motion. But a careful observation may indicate that the system is trying to recover some of the energy spend to overcome the aerodynamic drag. The concept of placing symmetrical turbines is presented for the very first time by us. We believe it requires more research and elaborate analysis which we expect to continue in future.

\section{References}

[1] Thomas D. Gillespie, "Fundamentals of Vehicle Dynamics", Society of Automotive Inc.

[2] Terry S. Boutet," Controlling Air Movements- A Manual for Builders and Architects", McGraw- Hill Book Company.

[3] Victor Olgyay, "Design with climate", Princeton University Press, 1963, p.104

[4] Godfrey Boyle, "Renewable Energy- Power for a sustainable future", Oxford University Press.

[5] Dr. Amalesh Chandra Mandal, Dr. Md. Quamrul Islam, "Aerodynamics and Design of Wind Turbines", Published by BUET.

[6] G.N.Tewari, A.K. Bansal, "Renewable Energy Resources", Narosa Publishing House.

[7] Martin O.L. Hansen, “Aerodynamics of Wind Turbines”, Earthscan, London.

[8] Bent Sorensen, "Renewable Energy" Academic Press, USA.

[9] Mukund R. Patel, "Wind and Solar Power Systems" CRC Press, USA.

[10] John D. Anderson, "Fundamentals of Aerodynamics", McGraw Hill Book Company. 